\title{
Nucleon electromagnetic form factors and polarization observables in space-like and time-like regions
}

\author{
Egle Tomasi-Gustafsson, F. Lacroix, C. Duterte and G. I. Gakh* \\ DAPNIA/SPhN, CEA/Saclay, 91191 Gif-sur-Yvette Cedex, France
}

(Dated: July 12, 2018)

\begin{abstract}
We perform a global analysis of the experimental data of the electromagnetic nucleon form factors, in space-like and time-like regions. We give the expressions of the observables in annihilation processes, such as $p+\bar{p} \rightarrow \ell^{+}+\ell^{-}, \ell=e$ or $\mu$, in terms of form factors. We discuss some of the phenomenological models proposed in the literature for the space-like region, and consider their analytical continuation to the time-like region. After determining the parameters through a fit on the available data, we give predictions for the observables which will be experimentally accessible with large statistics, polarized annihilation reactions.
\end{abstract}

PACS numbers: 13.40.Gp, 13.88.+e, 13.40.-f, 13.60.Fz

\section{INTRODUCTION}

Elastic hadron electromagnetic form factors (FFs) are fundamental quantities for the understanding of nucleon structure. They contain information on the nucleon ground state, and constitute a further severe test for the models of nucleon structure, which already reproduce the static properties of the nucleon, such as masses and magnetic moments. Moreover, the dependence of FFs on the momentum transfer squared, $q^{2}=-Q^{2}$, should reflect the transition from the non perturbative regime, where effective degrees of freedom describe the nucleon structure, to the asymptotic region, where QCD applies.

The magnetic proton $\mathrm{FF}$, which is the dominant term in the elastic ep cross section, has been measured at $Q^{2}$ values up to $31 \mathrm{GeV}^{2}$ in the space-like (SL) region [1], and from $p \bar{p}$ or $e^{+} e^{-}$annihilation up to $18 \mathrm{GeV}^{2}$ in the time-like (TL) region [2]. Large progress has been recently done in the determination of the electric and magnetic proton form factors, based on the idea, firstly suggested in Ref. [3], to measure the polarization of the recoil proton in $\overrightarrow{e p}$ elastic scattering, when the electron is longitudinally polarized.

Experiments, based on this method, have been performed at JLab up to $Q^{2}=5.6 \mathrm{GeV}^{2}[5,6]$. A similar method, applied to the reaction $d\left(e, e^{\prime} n\right) \mathrm{p}$ in quasi-elastic kinematics, has allowed the measurement of the neutron electric $\mathrm{FF}$ up to $Q^{2}=1 \mathrm{GeV}^{2}$ using a polarized deuteron target [7] and up to $Q^{2}=1.47 \mathrm{GeV}^{2}$, measuring the polarization of the outgoing neutron [8]. The polarization method has been also successfully applied at low $Q^{2}$, for a precise determination of the neutron FFs, at Mainz, and shows that $G_{E n}$ is definitely different from zero ([9] and refs therein).

These results have been obtained thanks to the availability of high intensity, highly polarized electron beams and polarized targets, and to the optimization of hadron polarimeters in the $\mathrm{GeV}$ range. An extension of the measurement of the polarization transfer in $\vec{e}+p \rightarrow e+\vec{p}$ up to $9 \mathrm{GeV}^{2}$ is in preparation [10].

More data are expected in future, in SL region, after the upgrade of Jlab, and in TL region, at Frascati and at the future FAIR facility at Darmstadt [4].

In the TL region [2], due to the poor statistics, the determination of FFs requires to integrate the differential cross section over a wide angular range. One typically assumes that the $G_{E}$ contribution plays a minor role in the cross section at large $q^{2}$ and the experimental results are usually given in terms of $\left|G_{M}\right|$, under the hypothesis that $G_{E}=0$ or $\left|G_{E}\right|=\left|G_{M}\right|$. The first hypothesis is an arbitrary one. The second hypothesis is strictly valid at threshold only, i.e., for $\tau=q^{2} /\left(4 m^{2}\right)=1$, but there is no theoretical argument which justifies its validity at any other momentum transfer, where $q^{2} \neq 4 m^{2}$ ( $m$ is the nucleon mass).

The measurement of the differential cross section for the process $p+\bar{p} \rightarrow \ell^{+}+\ell^{-}$at a fixed value of the total energy $s$, and for two different angles $\theta$, allowing the separation of the two FFs, $\left|G_{M}\right|^{2}$ and $\left|G_{E}\right|^{2}$, is equivalent to the well known Rosenbluth separation for the elastic ep-scattering. However, in TL region, this procedure is simpler, as it requires to change only one kinematical variable, $\cos \theta$, whereas, in SL region it is necessary to change simultaneously two kinematical variables: the energy of the initial electron and the electron scattering angle, fixing the momentum transfer squared, $Q^{2}$. Due to the limited statistics, the Rosenbluth separation of the $\left|G_{E}\right|^{2}$ and $\left|G_{M}\right|^{2}$ contributions has not yet been realized in TL region. Early attempts showed that the large error bars prevent to discriminate between the two hypothesis on $\left|G_{E}\right|$ and $\left|G_{M}\right|$ quoted above $[11,12]$.

\footnotetext{
* Permanent address:National Science Center KFTI, 310108 Kharkov, Ukraine
} 
The $\left|G_{M}\right|$ values depend, in principle, on the kinematics where the measurement was performed and the angular range of integration. However, it turns out that these two assumptions for $G_{E}$ lead to comparable values for $\left|G_{M}\right|$.

In the SL region the situation is different. The cross section for the elastic scattering of electrons on protons is sufficiently large to allow the measurements of the angular distribution and/or of polarization observables. Data on $G_{M}$ are available up to the highest measured value, $Q^{2} \simeq 31 \mathrm{GeV}^{2}[1]$ and this $\mathrm{FF}$ is often approximated according to a dipole behavior:

$$
G_{M}\left(Q^{2}\right) / \mu_{p}=G_{d}, \text { with } G_{d}=\left[1+Q^{2} / m_{d}^{2}\right]^{-2}, m_{d}^{2}=0.71 \mathrm{GeV}^{2},
$$

where $\mu_{p}$ is the magnetic moment of the proton.

It should be noted that the independent determination of both FFs, $G_{M}$ and $G_{E}$, from the unpolarized $e^{-}+p$-cross section, has been done up to $Q^{2}=8.7 \mathrm{GeV}^{2}[13]$, and the further extraction of $G_{M}$ assumes $G_{E}=G_{M} / \mu_{p}$. The behavior of $G_{E p}$, deduced from polarization experiments, in which, more precisely, the ratio $G_{E p} / G_{M p}$ is directly related to the longitudinal and transversal component of the scattered proton polarization, differs from $G_{M} / \mu_{p}$, with a deviation up to $70 \%$ at $Q^{2}=5.6 \mathrm{GeV}^{2}[6]$. This is the maximum momentum at which new, precise data are available.

The recent experimental data have inspired many new theoretical developments, and shown the necessity of a global representation of FFs in the full region of momentum transfer squared.

FFs are analytical functions of $q^{2}$, being real functions in the SL region (due to the hermiticity of the electromagnetic Hamiltonian) and complex functions in the TL region. The discussion of the constraints and consequences of a description in the full kinematical domain was firstly done in Ref. [14] and more recently in Refs. [15-18].

The extension of the nucleon models developed for the SL region to the TL region is straightforward for VMD inspired models, which may give a good description of all FFs in the whole kinematical region, after a fitting procedure involving a certain number of parameters [19, 20].

The purpose of this paper is to update and compare some of the available models on the world data set in both TL and SL regions, and to predict time-like polarization observables, in framework of these models. The paper is organized as follows. In section II the expressions for the relevant polarization observables, in the process $\bar{p}+p \rightarrow \ell^{+}+\ell^{-}$, $\ell=e$ or $\mu$, are given as a function of the electromagnetic FFs, in Section III we update some of the fits of nucleon FFs on the available data, and discuss their extension to the TL region. In section IV we give the predictions of the considered models in TL region.

\section{OBSERVABLES IN TL REGION}

We develop a simple and transparent formalism for the study of polarization phenomena for $p+\bar{p} \rightarrow \ell^{+}+\ell^{-}$, in framework of one-photon mechanism.

The calculations of the cross section and of the polarization observables for the process $\bar{p}+p \rightarrow \ell^{+}+\ell^{-}, \ell=e$ or $\mu$, in the annihilation channel are more conveniently performed in the center of mass system (CMS), Fig. 1. The momenta of the particles are indicated in the figure and $\left|\vec{k}_{1}\right|=\left|\vec{k}_{2}\right|=|\vec{k}|$ and $\left|\vec{p}_{1}\right|=\left|\vec{p}_{2}\right|=|\vec{p}|$. Let us choose the $z$ axis along the direction of the incoming antiproton, the $y$ axis normal to the scattering plane, and the $x$ axis to form a left-handed coordinate system. The components of the unity vectors are therefore $\hat{\vec{p}}=(0,0,1)$ and $\hat{\vec{k}}=(\sin \theta, 0, \cos \theta)$ with $\hat{\vec{p}} \cdot \hat{\vec{k}}=\cos \theta$, where $\theta$ is the electron production angle in CMS. The relevant kinematical variable is the antiproton energy, $E$, which is related to the four momentum transfer, $q^{2}=s=\left(k_{1}+k_{2}\right)^{2}=4 E^{2}$, as, in CMS, $\vec{k}_{1}+\vec{k}_{2}=0$. In the laboratory (Lab) system, one finds $q^{2}=2 m^{2}+2 m E_{L}$, where $E_{L}$ is the Lab antiproton energy. The observables are calculated in the approximation of zero electron mass.

The starting point of the analysis of the reaction $p+\bar{p} \rightarrow e^{+}+e-$ is the standard expression of the matrix element in framework of one-photon exchange mechanism:

$$
\mathcal{M}=\frac{e^{2}}{q^{2}} \bar{u}\left(-k_{2}\right) \gamma_{\mu} u\left(k_{1}\right) \bar{u}\left(p_{2}\right)\left[F_{1 N}\left(q^{2}\right) \gamma_{\mu}-\frac{\sigma_{\mu \nu} q_{\nu}}{2 m} F_{2 N}\left(q^{2}\right)\right] u\left(-p_{1}\right),
$$

where $p_{1}, p_{2}, k_{1}$ and $k_{2}$ are the four-momenta of initial antiproton and proton and the final electron and positron respectively, $q^{2}>4 m^{2}, q=k_{1}+k_{2}=p_{1}+p_{2}$. $F_{1 N}$ and $F_{2 N}$ are the Dirac and Pauli nucleon electromagnetic FFs, which are complex functions of the variable $q^{2}$ - in the TL region of momentum transfer.

In framework of one-photon exchange, the matrix element is written as the product of the leptonic and hadronic currents:

$$
\mathcal{M}=\frac{e^{2}}{q^{2}} \ell_{\mu} \mathcal{J}_{\mu}=\frac{e^{2}}{q^{2}}\left(\ell_{0} \mathcal{J}_{0}-\vec{\ell} \cdot \overrightarrow{\mathcal{J}}\right)=-\frac{e^{2}}{q^{2}} \vec{\ell} \cdot \overrightarrow{\mathcal{J}}
$$




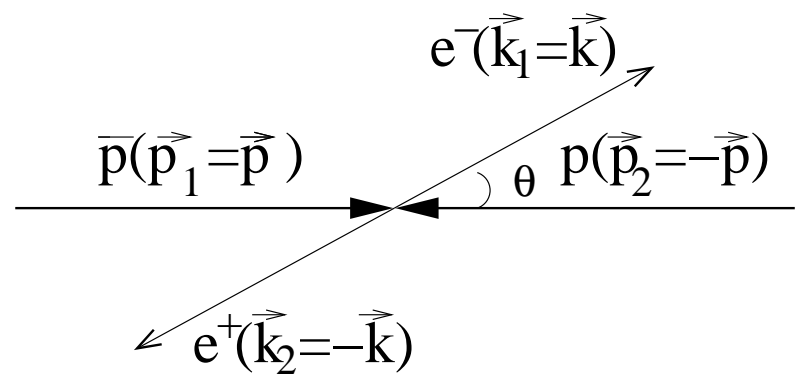

FIG. 1: The kinematics of the process $p+\bar{p} \rightarrow e^{-}+e^{+}$in the reaction CMS.

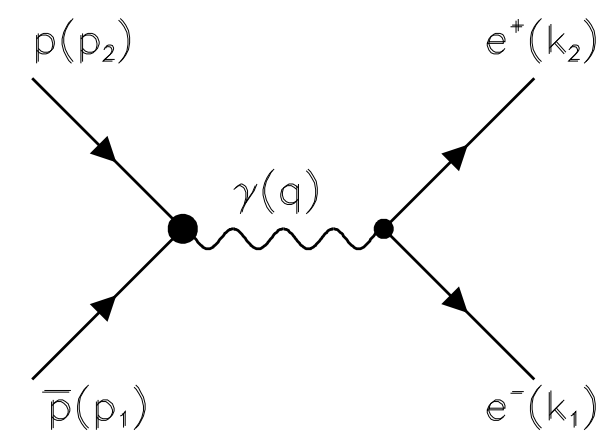

FIG. 2: One-photon mechanism for $p+\bar{p} \rightarrow e^{-}+e^{+}$(with notation of four particle four-momenta).

where $\ell_{0} \mathcal{J}_{0}=0$, due to the conservation of the leptonic and hadronic currents ${ }^{1}$. The expression for the leptonic current is:

$$
\vec{\ell}=\sqrt{q^{2}} \phi_{2}^{\dagger}(\vec{\sigma}-\hat{\vec{k}} \vec{\sigma} \cdot \hat{\vec{k}}) \phi_{1}
$$

where $\phi_{1}\left(\phi_{2}\right)$ is the two-component spinor of the electron (positron), $\hat{\vec{k}}$ is the unit vector along the final electron three-momentum, and for the hadronic current:

$$
\overrightarrow{\mathcal{J}}=\sqrt{q^{2}} \chi_{2}^{\dagger}\left[G_{M}\left(q^{2}\right)(\vec{\sigma}-\hat{\vec{p}} \vec{\sigma} \cdot \hat{\vec{p}})+\frac{1}{\sqrt{\tau}} G_{E}\left(q^{2}\right) \hat{\vec{p}} \vec{\sigma} \cdot \hat{\vec{p}}\right] \chi_{1},
$$

where $\chi_{1}$ and $\chi_{2}$ are the two-component spinors of the antiproton and the proton, $\hat{\vec{p}}$ is the unit vector along the three momentum of the antiproton in CMS.

From this expression one can see the physical meaning of the particular relation between the nucleon electromagnetic FFs at threshold:

$$
G_{E}\left(q^{2}\right)=G_{M}\left(q^{2}\right), q^{2}=4 m^{2} .
$$

The structure $\hat{\vec{p}} \vec{\sigma} \cdot \hat{\vec{p}}$ describes the $\bar{p}+p$ annihilation from $D$-wave, i.e., with angular momentum $\ell=2$. At threshold, where $\tau \rightarrow 1$, the finite radius of the strong interaction allows only the S-state, and $G_{M}\left(q^{2}\right)-\frac{1}{\sqrt{\tau}} G_{E}\left(q^{2}\right)=0$.

\footnotetext{
1 The conservation of the current implies that $\ell \cdot q=0$, i.e., $\ell_{0} q_{0}-\vec{\ell} \cdot \vec{q}=0$, but $\vec{q}=\vec{k}_{1}+\vec{k}_{2}=0$ in CMS. Therefore, $\ell_{0} q_{0}=0$ for any energy $q_{0}$, i.e., $\ell_{0}=0$.
} 
From Eqs. (3), (4), and (5) one can find the formulas for the unpolarized cross section, the angular asymmetry and all the polarization observables.

\section{A. The cross section}

To calculate the cross section when all particles are unpolarized, one has to sum over the polarization of the final particles and to average over the polarization of initial particles:

$$
\begin{gathered}
\left(\frac{d \sigma}{d \Omega}\right)_{0}=\frac{|\overline{\mathcal{M}}|^{2}}{64 \pi^{2} q^{2}} \frac{k}{p}, k=\frac{\sqrt{\left(q^{2}\right)}}{2}, p=\sqrt{\frac{\left(q^{2}\right)}{4}-m^{2}} \\
|\overline{\mathcal{M}}|^{2}=\frac{1}{4} \frac{e^{4}}{q^{4}} \ell_{a b} \mathcal{J}_{a b}, \ell_{a b}=\ell_{a} \ell_{b}^{*}, \mathcal{J}_{a b}=\mathcal{J}_{a} \mathcal{J}_{b}^{*}
\end{gathered}
$$

Using the expressions (4) and (5), the formula for the cross section in CMS is:

$$
\left(\frac{d \sigma}{d \Omega}\right)_{0}=\mathcal{N}\left[\left(1+\cos ^{2} \theta\right)\left|G_{M}\right|^{2}+\frac{1}{\tau} \sin ^{2} \theta\left|G_{E}\right|^{2}\right]
$$

where $\mathcal{N}=\frac{\alpha^{2}}{4 \sqrt{q^{2}\left(q^{2}-4 m^{2}\right)}}, \alpha=e^{2} /(4 \pi) \simeq 1 / 137$, is a kinematical factor. This formula was firstly obtained in Ref. $[21]$.

The angular dependence of the cross section, Eq. (7), results directly from the assumption of one-photon exchange, where the photon has spin 1 and the electromagnetic hadron interaction satisfies the $P$-invariance. Therefore, the measurement of the differential cross section at three angles (or more) would also allow to test the presence of $2 \gamma$ exchange [22].

The electric and the magnetic FFs are weighted by different angular terms, in the cross section, Eq. (7). One can define an angular asymmetry, $\mathcal{R}$, with respect to the differential cross section measured at $\theta=\pi / 2, \sigma_{0}[15]$ :

$$
\left(\frac{d \sigma}{d \Omega}\right)_{0}=\sigma_{0}\left[1+\mathcal{R} \cos ^{2} \theta\right]
$$

where $\mathcal{R}$ can be expressed as a function of FFs:

$$
\mathcal{R}=\frac{\tau\left|G_{M}\right|^{2}-\left|G_{E}\right|^{2}}{\tau\left|G_{M}\right|^{2}+\left|G_{E}\right|^{2}} .
$$

This observable should be very sensitive to the different underlying assumptions on FFs, therefore, a precise measurement of this quantity, which does not require polarized particles, would be very interesting.

The $q^{2}$ dependence of the total cross section can be presented as follows:

$$
\sigma\left(q^{2}\right)=\mathcal{N} \frac{8}{3} \pi\left[2\left|G_{M}\right|^{2}+\frac{1}{\tau}\left|G_{E}\right|^{2}\right] .
$$

Polarization phenomena will be especially important in $p+\bar{p} \rightarrow \ell^{+}+\ell^{-}$. The dependence of the cross section on the polarizations $\vec{P}_{1}$ and $\vec{P}_{2}$ of the colliding antiproton and proton can be written as follows:

$$
\begin{aligned}
\left(\frac{d \sigma}{d \Omega}\right)_{0}\left(\vec{P}_{1}, \vec{P}_{2}\right)= & \left(\frac{d \sigma}{d \Omega}\right)_{0}\left[1+A_{y}\left(P_{1 y}+P_{2 y}\right)+A_{x x} P_{1 x} P_{2 x}+A_{y y} P_{1 y} P_{2 y}+A_{z z} P_{1 z} P_{2 z}\right. \\
& \left.+A_{x z}\left(P_{1 x} P_{2 z}+P_{1 z} P_{2 x}\right)\right],
\end{aligned}
$$

where the coefficients $A_{i}$ and $A_{i j}(i, j=x, y, z)$, analyzing powers and correlation coefficients, depend on the nucleon FFs. Their explicit form is given in the following sections. The dependence (11) results from the P-invariance of hadron electrodynamics. 


\section{B. Single spin polarization observables}

In case of polarized antiproton beam with polarization $\vec{P}_{1}$, the contribution to the cross section can be calculated as:

$$
\left(\frac{d \sigma}{d \Omega}\right)_{0} \overrightarrow{A_{1}}=-\ell_{a b} \frac{1}{4} \operatorname{Tr} \mathcal{J}_{a} \vec{\sigma} \mathcal{J}_{b}^{*}
$$

Here the terms related to $\left|G_{E}\right|^{2}$ and $\left|G_{M}\right|^{2}$ vanish. For the interference terms, the only non zero analyzing power is related to $P_{y}$ :

$$
\left(\frac{d \sigma}{d \Omega}\right)_{0} A_{1, y}=\frac{\mathcal{N}}{\sqrt{\tau}} \sin 2 \theta \operatorname{Im}\left(G_{M} G_{E}^{*}\right)
$$

When the target is polarized, one writes:

$$
\left(\frac{d \sigma}{d \Omega}\right)_{0} \vec{A}_{2}=\ell_{a b} \frac{1}{4} \operatorname{Tr} \mathcal{J}_{a} \mathcal{J}_{b}^{*} \vec{\sigma}
$$

Again the terms related to $\left|G_{E}\right|^{2}$ and $\left|G_{M}\right|^{2}$ vanish. Moreover, one can find $\overrightarrow{A_{2}}=\overrightarrow{A_{1}}=\vec{A}$.

Eq. (13) has been proved also in Ref. [21]. One can see that this analyzing power, being T-odd, does not vanish in $p+\bar{p} \rightarrow \ell^{+}+\ell^{-}$, even in one-photon approximation, due to the fact FFs are complex in time-like region. This is a principal difference with elastic ep scattering. Let us note also that the assumption $G_{E}=G_{M}$ implies $A_{y}=0$, independently from any model taken for the calculation of FFs.

\section{Double spin polarization observables}

The contribution to the cross section, when both colliding particles are polarized is calculated through the following expression:

$$
\left(\frac{d \sigma}{d \Omega}\right)_{0} A_{a b}=-\frac{1}{4} \ell_{m n} \operatorname{Tr} \mathcal{J}_{m} \sigma_{a} \mathcal{J}_{n}^{\dagger} \sigma_{b}
$$

where $a$ and $b=x, y, z$ refer to the $a(b)$ component of the projectile (target) polarization. Among the nine possible terms, $A_{x y}=A_{y x}=A_{z y}=A_{y z}=0$, and the nonzero components are:

$$
\begin{aligned}
& \left(\frac{d \sigma}{d \Omega}\right)_{0} A_{x x}=\sin ^{2} \theta\left(\left|G_{M}\right|^{2}+\frac{1}{\tau}\left|G_{E}\right|^{2}\right) \mathcal{N}, \\
& \left(\frac{d \sigma}{d \Omega}\right)_{0} A_{y y}=-\sin ^{2} \theta\left(\left|G_{M}\right|^{2}-\frac{1}{\tau}\left|G_{E}\right|^{2}\right) \mathcal{N}, \\
& \left(\frac{d \sigma}{d \Omega}\right)_{0} A_{z z}=\left[\left(1+\cos ^{2} \theta\right)\left|G_{M}\right|^{2}-\frac{1}{\tau} \sin ^{2} \theta\left|G_{E}\right|^{2}\right] \mathcal{N}, \\
& \left(\frac{d \sigma}{d \Omega}\right)_{0} A_{x z}=\left(\frac{d \sigma}{d \Omega}\right)_{0} A_{z x}=\frac{1}{\sqrt{\tau}} \sin 2 \theta \operatorname{Re} G_{E} G_{M}^{*} \mathcal{N} .
\end{aligned}
$$

One can see that the double spin observables depend on the moduli squared of FFs, besides $A_{x z}$. Therefore, in order to determine the relative phase of FFs, in TL region, the interesting observables are $A_{y}$, and $A_{x z}$ which contain, respectively, the imaginary and the real part of the product $G_{E} G_{M}^{*}$.

\section{RESULTS AND DISCUSSION}

\section{A. The data}

The nucleon FFs world data were collected and listed in Table I for proton FFs and Table II for neutron FFs in SL region. 
In Fig. 3 the nucleon FFs world data in SL region are shown: the ratio $\mu_{p} G_{E p} / G_{M p}$ (Fig. 3a), the magnetic proton FF normalized to the dipole FF and divided by $\mu_{p}$ (Fig. 3b), the electric neutron FF (Fig. 3c), and the magnetic neutron FF normalized to the dipole FF and divided by $\mu_{n}$ (Fig. 3d). For the electric proton FF, the discrepancy among the data measured with the Rosenbluth methods (stars) and the polarization method (solid squares) appears clearly in Fig. 3a. This problem has widely been discussed in the literature, (for a recent discussion see, for instance, Ref. [23]) and rises fundamental issues. If the trend indicated by polarization measurements is confirmed at higher $Q^{2}$ [10], not only the electric and magnetic charge distribution in the nucleus are different and deviate, classically, from an exponential charge distribution, but also the electric FF has a zero and becomes eventually negative. This scenario will change our view on the nucleon structure and will favor VMD inspired models like [19, 20], which can reproduce such behavior.

We included data issued from both kind of measurements in the fit, although if a consensus seems to appear that FFs extracted from polarization measurements are more reliable, as less affected by all kinds of radiative corrections. Our purpose here is not to get the best $\chi^{2}$, but to get a global description of the overall data. The precision and the number of points is very different for the different FFs, therefore one can obtain a good $\chi^{2}$ for a model that reproduces well, for example, the electric and magnetic FFs in the SL region and fails in giving the trend of $G_{E n}$ in TL region.

We included in the fit the data on proton magnetic FFs which were published after 1973, and we did not include the data on the neutron electric FFs from Refs. [35, 36, 38, 41, 44] as data of much better precision were, later, available, in the same $Q^{2}$ range.

The data in the TL region are drawn in In Fig. 4a, b for the proton and in Fig. 4c, $\mathrm{d}$ for the neutron, respectively and summarized in Table III. As no separation has been done for electric and magnetic FFs, the data are extracted under the hypothesis that $\left|G_{E N}\right|=\left|G_{M N}\right|$. Concerning the neutron, the first and still unique measurement was done at Frascati, by the collaboration FENICE [45].

\section{B. The models}

Among the existing models of nucleon FFs, we consider some parametrizations, which have an analytical expression that can be continued in TL region: predictions of pQCD, in a form generally used as simple fit to experimental data, a model based on vector meson dominance (VMD) [62], and a third model based on an extension of VMD, with additional terms in order to satisfy the asymptotic predictions of QCD [63], in the form called GKex(02L). We also considered the Hohler parametrization [64] and the Bosted empirical fit [65].

In order to help the reader, we report in the Appendix the explicit forms of the parametrizations previously published, with the parameters corresponding to the present fit, compared to the published ones.

The pQCD prediction, based on counting rules, follows the dipole behavior (1) in SL region, and can be extended in TL region as [66]:

$$
\left|G_{M}\right|=\frac{A(N)}{q^{4} \ln ^{2}\left(q^{2} / \Lambda^{2}\right)},
$$

where $\Lambda=0.3 \mathrm{GeV}$ is the QCD scale parameter and $A$ is a free parameter. This simple parametrization is taken to be the same for proton and neutron. The best fit ( Fig. 4, dashed line) is obtained with a parameter $A(p)=56.3$ $\mathrm{GeV}^{4}$ for proton and $A(n)=77.15 \mathrm{GeV}^{4}$ for neutron, which reflects the fact that in TL region, neutron FFs are larger than for proton. One should note that errors are also larger in TL region.

A possible explanation of the fact that FFs are systematically larger in TL region than in SL region (which is true also in the proton case) is the presence of a resonance in the $N \bar{N}$ system, just below the $N \bar{N}$ threshold [67].

More pQCD inspired parametrizations exist for the form factor ratio $F_{2} / F_{1}$, which include logarithmic corrections, and have been recently discussed in Ref. [16]. However, some of these analytical forms have problems related to the asymptotic behavior. This will be discussed in a future paper.

The analytical continuation to TL region of the other models is based on the following relations:

$$
Q^{2}=-q^{2}=q^{2} e^{-i \pi} \Longrightarrow\left\{\begin{array}{c}
\ln \left(Q^{2}\right)=\ln \left(q^{2}\right)-i \pi \\
\sqrt{Q^{2}}=e^{\frac{-i \pi}{2}} \sqrt{q^{2}}
\end{array}\right.
$$

Most of the models predict a different behavior for the electric and the magnetic FFs in TL region, whereas, as already mentioned, no individual determination of electric and magnetic FFs has been done yet. We chose to fit the data assuming that they correspond to the magnetic FFs for proton and neutron, Fig. 4a and 4c, respectively. Therefore, the curves for the electric FFs, in Figs. 4b and 4d have to be considered predictions from the models. Including or not the data on neutron FFs, in TL region, influence very little the fitting procedure. 
The parametrization from Ref. [62] is shown as a dotted line, in Figs. 3 and 4. This model is based on a view of the nucleon as composed by an inner core with a small radius (described by a dipole term) surrounded by a meson cloud. While it reproduces very well the proton data in SL region (and particularly the polarization measurements), it fails in reproducing the large $Q^{2}$ behaviour of the magnetic neutron FF in SL region. The present fit constrained on the TL data and on the recent SL data does not improve the situation. In framework of this model a good global fit in SL region has been obtained with a modification including a phase in the common dipole term. However, the TL region is less well reproduced [20]. Therefore, the curves drawn in all the figures correspond to the original parameters, which give, in our opinion, a better representation of the whole set of data.

The result from an update fit based on the parametrization GKex(02L) [63] is shown in Figs. 3 and 4 (solid line). It is possible to find a good overall parametrization, with parameters not far from those found in the original paper for the SL region only. The agreement is very good, for both proton and neutron FFs.

The Hohler parametrization [64], contains also pole terms with adjustable parameters. The $\rho$-exchange contribution, however, is fully determined, with constants fixed on $\pi N$ data. The model contains 17 parameters, already, so we did not try to readjust or refit the $\rho$-contribution. As noted in the original paper, such model is not suited to the extrapolation to TL region, because poles appear in the physical region. Constraining the parameters, in order to avoid these instabilities, worsens the description in the SL region. Therefore, we give only a fit on all FFs, in SL region, Fig. 3 (dash-dotted line), corresponding to $\chi^{2} / n d f \simeq 1$. . The formulas as well as the original and updated parameters are also given in Appendix. Parametrization [19] can be considered a successful generalization, in TL region, based on unitarity and analyticity. It requires the modelization of ten resonances, five isoscalar and five isovector.

The Bosted parametrization [65] is an empirical fit to nucleon FFs, in the SL region, based on simple formulas which are useful for fast estimations. It does not seem possible to find a unique function, which describes satisfactorily both the magnetic nucleon FFs and the electric proton FF, so the parameters are specific to each FFs. In the extension to TL region, as for the Hohler parametrization, one can not avoid poles and instabilities, and attempts to obtain a description in SL and TL regions remained unsuccessful. Therefore, we give the fit for the SL region, only, as dashed line in Fig. 3, and report in the Appendix the useful formulas and the updated parameters. As one can see from the table, they do not differ more than $20 \%$ from the published ones and the fit corresponds to $\chi^{2} / n d f \simeq 2$.

\section{PREDICTIONS IN TL REGION}

We give the predictions for the cross section asymmetry and the polarization observables, for those models, described above, which give a good overall description of the available FFs data in SL and TL regions. The calculation is based on Eqs. (8), (13) and (14), for a fixed value of the angle $\theta=\pi / 4$.

As shown in Fig. 5, all these observables are, generally, quite large. The model [62] predicts the largest (absolute) value at $q^{2} \simeq 15 \mathrm{GeV}^{2}$ for all observables, except $A_{x z}$, which has two pronounced extrema.

All observables manifest a different behavior, according to the different models. The sign, also, can be opposite for VMD inspired models and pQCD. The model [63] is somehow intermediate between the two representations, as it contains the asymptotic predictions of QCD (at the expenses of a large number of parameters).

The fact that single spin observables in annihilation reactions are discriminative towards models, especially at threshold, was already pointed out in Ref. [69], for the process $e^{+}+e^{-} \rightarrow p+\bar{p}$ on the basis of two versions of a unitary VDM model. The present results, (Fig. 5), for the inverse reaction $p+\bar{p} \rightarrow e^{+}+e^{-}$confirm this trend and show that experimental data will be extremely useful, particularly in the kinematical region around $q^{2} \simeq 15 \mathrm{GeV}{ }^{2}$.

\section{SUMMARY}

The measurement of polarization observables and the possibility to access individual nucleon FFs in TL and SL regions at larger $Q^{2}$ and/or with higher precision is foreseen in next future.

A general analysis of the experimental data on nucleon electromagnetic FFs, extracted from elastic scattering and annihilation reactions, has been performed in the available kinematical region.

Expressions of the experimental observables in the reaction $p+\bar{p} \rightarrow e^{+}+e^{-}$have been derived in terms of the electromagnetic FFs, as a function of the momentum transfer squared.

Some of the models on nucleon FFs have been reviewed, extended in TL region and used to give predictions on experimental observables which should be useful to plan future experiments.

Many questions are still open. Recent data in the SL region show that the ratio $G_{E p} / G_{M p}$ deviates from the expected dipole behavior. In the TL region, the values of $\left|G_{M}\right|$, obtained under the assumption that $G_{E}=G_{M}$, are larger than the corresponding SL values. This has been considered as a proof of the non applicability of the 
Phràgmen-Lindelöf theorem, (up to $s=18 \mathrm{GeV}^{2}$, at least) or as an evidence that the asymptotic regime is not reached [14]. The presence of a large relative phase of magnetic and electric proton FFs in the TL region, if experimentally proved at relatively large momentum transfer, can be considered a strong indication that these FFs have a different behavior. In particular, it will allow a test of the Phràgmen-Lindelöf theorem [14].

Large progress in view of a global interpretation of the nucleon FFs is expected from future experiments with antiproton beams: it will be possible, at the future FAIR facility at GSI, to separate the electric and magnetic FFs in a wide region of $s$ and to extend the measurement of FFs up to the largest available energy, corresponding to $s \simeq 30$ $\mathrm{GeV}^{2}$.

The angular distribution of the produced leptons will allow the separation of the electric and magnetic FFs. The measurement of the asymmetry $\mathcal{R}$ (from the angular dependence of the differential cross section for $p+\bar{p} \leftrightarrow \ell^{+}+\ell^{-}$) is sensitive to the relative value of $\left|G_{M}\right|$ and $\left|G_{E}\right|$. In particular, the $\theta$-dependence of the single spin and double spin polarization observables is very sensitive to existing models of the nucleon FFs, which reproduce equally well the data in SL region.

Similar information can be obtained from the final polarization in $\ell^{+}+\ell^{-} \rightarrow \vec{p}+\bar{p}[69]$, but in this case one has to deal with the problem of hadron polarimetry, in conditions of very small cross sections.

Only the study of the processes $p+\bar{p} \rightarrow \pi^{0}+\ell^{+}+\ell^{-}$and $p+\bar{p} \rightarrow \pi^{+}+\pi^{-}+\ell^{+}+\ell^{-}$, [70, 71] will allow to measure proton FFs in the unphysical region (for $s \leq 4 m^{2}$, where the vector meson contribution plays an important role) and to determine the relative phase of pion and nucleon FFs.

\section{APPENDIX}

The Sachs FFs are expressed in terms of the Pauli and Dirac FFs as:

$$
G_{E}^{N}=F_{1}^{N}\left(Q^{2}\right)+\tau F_{2}^{N}\left(Q^{2}\right), G_{M}^{N}=F_{1}^{N}\left(Q^{2}\right)+F_{2}^{N}\left(Q^{2}\right)
$$

One can introduce the isoscalar and isovector FFs $F_{i}^{s}$ and $F_{i}^{v}, i=1,2$ as: $2 F_{i}^{p}=F_{i}^{s}+F_{i}^{v}, 2 F_{i}^{n}=F_{i}^{s}-F_{i}^{v}$.

Then, the isoscalar and isovector currents can be parametrized in terms of meson propagators, effective FFs, and/or terms which insure specific properties, according to the different models.

\section{A. Model from Iachello, Jackson and Landé [62] and Iachello and Wan [18]}

FFs are parametrized following the work [62], with a modification that consists in adding a phase in the dipole term, $g\left(Q^{2}\right)$, for the extension in TL region.

$$
\begin{aligned}
& F_{1}^{s}\left(Q^{2}\right)=\frac{g\left(Q^{2}\right)}{2}\left[\left(1-\beta_{\omega}-\beta_{\phi}\right)+\beta_{\omega} \frac{\mu_{\omega}^{2}}{\mu_{\omega}^{2}+Q^{2}}+\beta_{\phi} \frac{\mu_{\phi}^{2}}{\mu_{\phi}^{2}+Q^{2}}\right], \\
& F_{1}^{v}\left(Q^{2}\right)=\frac{g\left(Q^{2}\right)}{2}\left[\left(1-\beta_{\rho}\right)+\beta_{\rho} \frac{\mu_{\rho}^{2}+8 \Gamma_{\rho} \mu_{\pi} / \pi}{\left(\mu_{\rho}^{2}+Q^{2}\right)+\left(4 \mu_{\pi}^{2}+Q^{2}\right) \Gamma_{\rho} \alpha\left(Q^{2}\right) / \mu_{\pi}}\right], \\
& F_{2}^{s}\left(Q^{2}\right)=\frac{g\left(Q^{2}\right)}{2}\left[\left(\mu_{p}+\mu_{n}-1-\alpha_{\phi}\right) \frac{\mu_{\omega}^{2}}{\mu_{\omega}^{2}+Q^{2}}+\alpha_{\phi} \frac{\mu_{\phi}^{2}}{\mu_{\phi}^{2}+Q^{2}}\right], \\
& F_{2}^{v}\left(Q^{2}\right)=\frac{g\left(Q^{2}\right)}{2}\left[\left(\mu_{p}-\mu_{n}-1\right) \frac{\mu_{\rho}^{2}+8 \Gamma_{\rho} \mu_{\pi} / \pi}{\left(\mu_{\rho}^{2}+Q^{2}\right)+\left(4 \mu_{\pi}^{2}+Q^{2}\right) \Gamma_{\rho} \alpha\left(Q^{2}\right) / \mu_{\pi}}\right],
\end{aligned}
$$

with $g\left(Q^{2}\right)=\frac{1}{\left(1+\gamma e^{i \theta} Q^{2}\right)^{2}}$ and $\alpha\left(Q^{2}\right)=\frac{2}{\pi} \sqrt{\frac{Q^{2}+4 \mu_{\pi}^{2}}{Q^{2}}} \ln \left[\frac{\sqrt{\left(Q^{2}+4 \mu_{\pi}^{2}\right)}+\sqrt{Q^{2}}}{2 \mu_{\pi}}\right]$, with the standard values of the masses $m=0.939 \mathrm{GeV}, \mu_{\rho}=0.77 \mathrm{GeV}, \mu_{\omega}=0.78 \mathrm{GeV}, \mu_{\phi}=1.02 \mathrm{GeV}, \mu_{\pi}=0.139 \mathrm{GeV}$ and the $\rho$ width $\Gamma_{\rho}=0.112 \mathrm{GeV}$.

The values of the six parameters are given in Table V. 


\section{B. Model from Lomon [63]}

$$
\begin{aligned}
& F_{1}^{v}\left(Q^{2}\right)=\frac{N}{2}\left[\frac{1.0317+0.0875\left(1+Q^{2} / 0.3176\right)^{-2}}{\left(1+Q^{2} / 0.5496\right)}+\frac{g_{\rho^{\prime}}}{f_{\rho^{\prime}}} \frac{m_{\rho^{\prime}}^{2}}{m_{\rho^{\prime}}^{2}+Q^{2}}\right] F_{1}^{\rho}\left(Q^{2}\right)+ \\
& \left(1-1.1192 \frac{N}{2}-\frac{g_{\rho^{\prime}}}{f_{\rho^{\prime}}}\right) F_{1}^{D}\left(Q^{2}\right), \\
& F_{2}^{v}\left(Q^{2}\right)=\frac{N}{2}\left[\frac{5.7824+0.3907\left(1+Q^{2} / 0.1422\right)^{-1}}{\left(1+Q^{2} / 0.5362\right)}+\kappa_{\rho^{\prime}} \frac{g_{\rho^{\prime}}}{f_{\rho^{\prime}}} \frac{m_{\rho^{\prime}}^{2}}{m_{\rho^{\prime}}^{2}+Q^{2}}\right] F_{2}^{\rho}\left(Q^{2}\right)+ \\
& \left(\kappa_{\nu}-6.1731 \frac{N}{2}-\kappa_{\rho^{\prime}} \frac{g_{\rho^{\prime}}}{f_{\rho^{\prime}}}\right) F_{2}^{D}\left(Q^{2}\right), \\
& F_{1}^{s}\left(Q^{2}\right)=\left(\frac{g_{\omega}}{f_{\omega}} \frac{m_{\omega}^{2}}{m_{\omega}^{2}+Q^{2}}+\frac{g_{\omega^{\prime}}}{f_{\omega^{\prime}}} \frac{m_{\omega^{\prime}}^{2}}{m_{\omega^{\prime}}^{2}+Q^{2}}\right) F_{1}^{\omega}\left(Q^{2}\right)+ \\
& \frac{g_{\phi}}{f_{\phi}} \frac{m_{\phi}^{2}}{m_{\phi}^{2}+Q^{2}} F_{1}^{\phi}\left(Q^{2}\right)+\left(1-\frac{g_{\omega}}{f_{\omega}}-\frac{g_{\omega^{\prime}}}{f_{\omega^{\prime}}}\right) F_{1}^{D}\left(Q^{2}\right), \\
& F_{2}^{s}\left(Q^{2}\right)=\left(\kappa_{\omega} \frac{g_{\omega}}{f_{\omega}} \frac{m_{\omega}^{2}}{m_{\omega}^{2}+Q^{2}}+\kappa_{\omega^{\prime}} \frac{g_{\omega^{\prime}}}{f_{\omega^{\prime}}} \frac{m_{\omega^{\prime}}^{2}}{m_{\omega^{\prime}}^{2}+Q^{2}}\right) F_{2}^{\omega}\left(Q^{2}\right)+\kappa_{\phi} \frac{g_{\phi}}{f_{\phi}} \frac{m_{\phi}^{2}}{m_{\phi}^{2}+Q^{2}} F_{2}^{\phi}\left(Q^{2}\right)+ \\
& \left(\kappa_{s}-\kappa_{\omega} \frac{g_{\omega}}{f_{\omega}}-\kappa_{\omega^{\prime}} \frac{g_{\omega^{\prime}}}{f_{\omega^{\prime}}}-\kappa_{\phi} \frac{g_{\phi}}{f_{\phi}}\right) F_{2}^{D}\left(Q^{2}\right),
\end{aligned}
$$

with

$$
\begin{aligned}
F_{1}^{\alpha, D}\left(Q^{2}\right) & =\frac{\Lambda_{1, D}^{2}}{\Lambda_{1, D}^{2}+\widetilde{Q}^{2}} \frac{\Lambda_{2}^{2}}{\Lambda_{2}^{2}+\widetilde{Q}^{2}}, \alpha=\rho, \omega \text { and } \Lambda_{1, D} \equiv \Lambda_{1} \text { for } F_{i}^{\alpha}, \Lambda_{1, D} \equiv \Lambda_{D} \text { for } F_{i}^{D} \\
F_{2}^{\alpha, D}\left(Q^{2}\right) & =\frac{\Lambda_{1, D}^{2}}{\Lambda_{1, D}^{2}+\widetilde{Q}^{2}}\left(\frac{\Lambda_{2}^{2}}{\Lambda_{2}^{2}+\widetilde{Q}^{2}}\right)^{2}, F_{1}^{\phi}\left(Q^{2}\right)=F_{1}^{\alpha}\left(\frac{Q^{2}}{\Lambda_{1}^{2}+Q^{2}}\right)^{1.5}, \\
F_{2}^{\phi}\left(Q^{2}\right) & =F_{2}^{\alpha}\left(\frac{\Lambda_{1}^{2}}{\mu_{\phi}^{2}} \frac{Q^{2}+\mu_{\phi}^{2}}{\Lambda_{1}^{2}+Q^{2}}\right)^{1.5}, \widetilde{Q}^{2}=Q^{2} \frac{\ln \left[\left(\Lambda_{D}^{2}+Q^{2}\right) / \Lambda_{Q C D}^{2}\right]}{\ln \left(\Lambda_{D}^{2} / \Lambda_{Q C D}^{2}\right)} .
\end{aligned}
$$

The set of parameters is reported in Table IV.

\section{Model from Hohler [64]}

This model is also based on a VMD parametrization:

$$
\begin{aligned}
F_{1}^{\rho}\left(Q^{2}\right) & =0.5\left[0.955+\frac{0.09}{\left(1+Q^{2} / 0.355\right)^{2}}\right] \frac{1}{1+Q^{2} / 0.536} \\
F_{2}^{\rho}\left(Q^{2}\right) & =0.5\left[5.335+\frac{0.962}{\left(1+Q^{2} / 0.268\right)^{2}}\right] \frac{1}{1+Q^{2} / 1.603} \\
F_{i}^{(s)}\left(Q^{2}\right) & =\sum_{j} \frac{a_{j}^{(i, s)}}{b_{j}^{(s)}+Q^{2}} \\
F_{i}^{(v)}\left(Q^{2}\right) & =F_{i}^{\rho}\left(Q^{2}\right)+\sum_{j} \frac{a_{j}^{(i, v)}}{b_{j}^{(v)}+Q^{2}} .
\end{aligned}
$$

The parameters are given in Table VI. 


\section{Model from Bosted [65]}

The analytical expressions are inverse of polynomes as functions of $Q$, whereas $G_{E n}$ is described by a different function, as suggested by Galster [68]:

$$
\begin{aligned}
F^{j} & =\frac{1}{1+\sum_{i} a_{i}^{j} Q^{i}}, \\
G_{E}^{n} & =\frac{\alpha \mu_{n} \tau G_{D}\left(Q^{2}\right)}{1+\beta \tau},
\end{aligned}
$$

with $a_{i}^{j}, \alpha$ and $\beta$ free parameters. In the present notation $j=1,2,3$ corresponds to $G_{E p}$ and $G_{M n}$ and $G_{M p}$, respectively. The inverse polynomes are of fourth order for $G_{E p}$ and $G_{M n}$ and of fifth order for $G_{M p}$.

The parameters are given in Table VII.

a

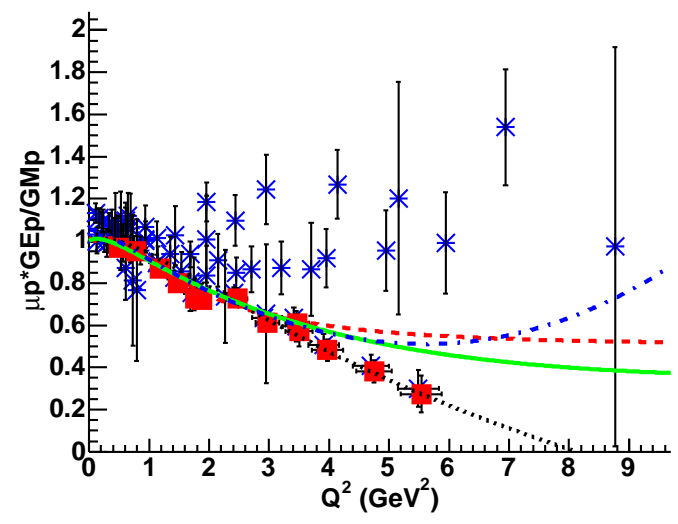

c

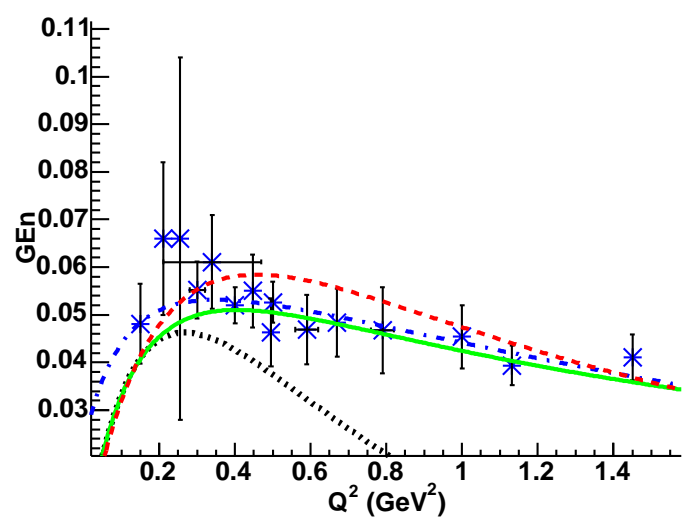

b

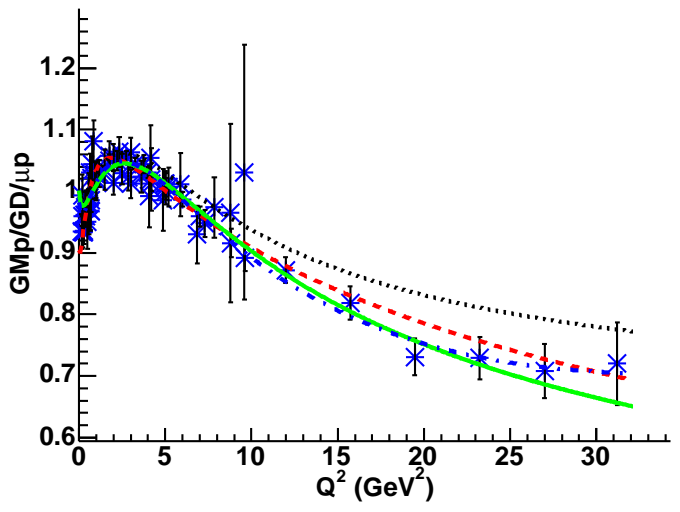

d

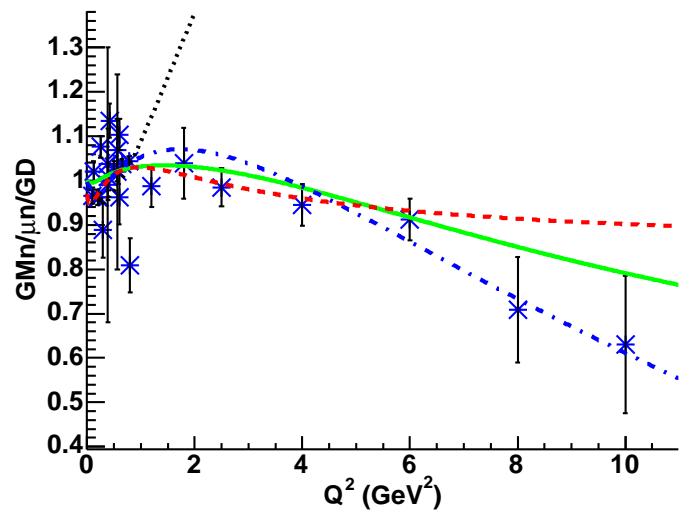

FIG. 3: Nucleon Form Factors in Space-Like region: (a) proton electric FF, scaled by $\mu_{p} G_{M p}$ (b) proton magnetic FF scaled by $\mu_{p} G_{D}$, (c) neutron electric FF, (d) neutron magnetic FF, scaled by $\mu_{n} G_{D}$. The predictions of the models are drawn: from Ref. [62] (dotted line), from Ref. [63] (solid line), model from Ref. Ref. [64] (dash-dotted line), from [65] (dashed line).

[1] R. G. Arnold et al., Phys. Rev. Lett. 57, 174 (1986).

[2] M. Andreotti et al., Phys. Lett. B 559, 20 (2003) and refs herein.

[3] A. Akhiezer and M. P. Rekalo, Dokl. Akad. Nauk USSR, 180, 1081 (1968); Sov. J. Part. Nucl. 4, 277 (1974); M. P. Rekalo and E. Tomasi-Gustafsson, Lecture Notes, arXiv:nucl-th/0202025. 
a

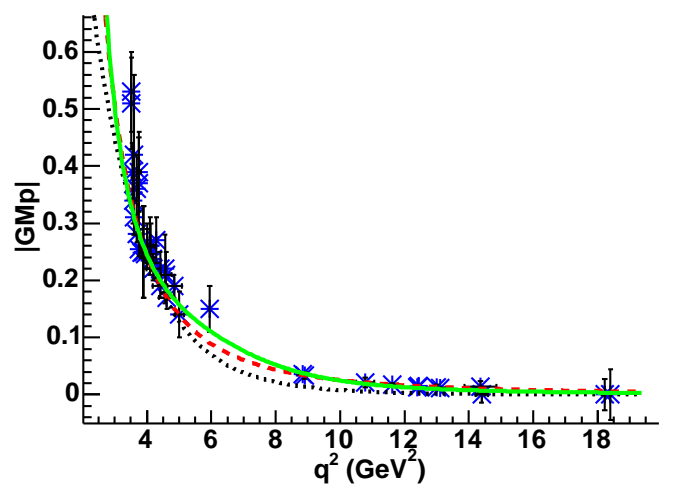

c

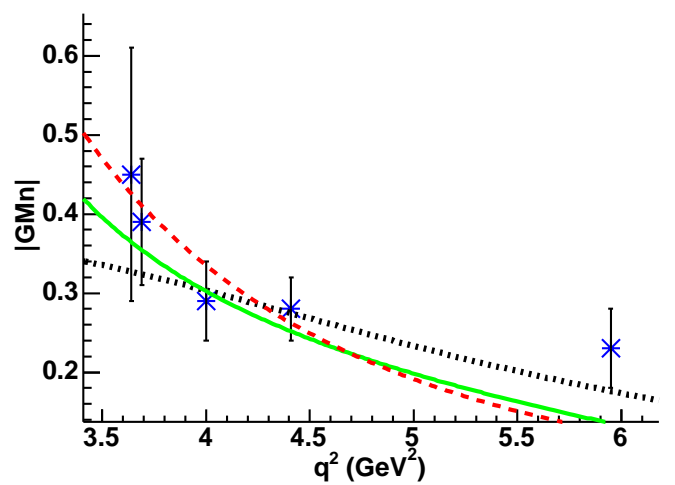

b

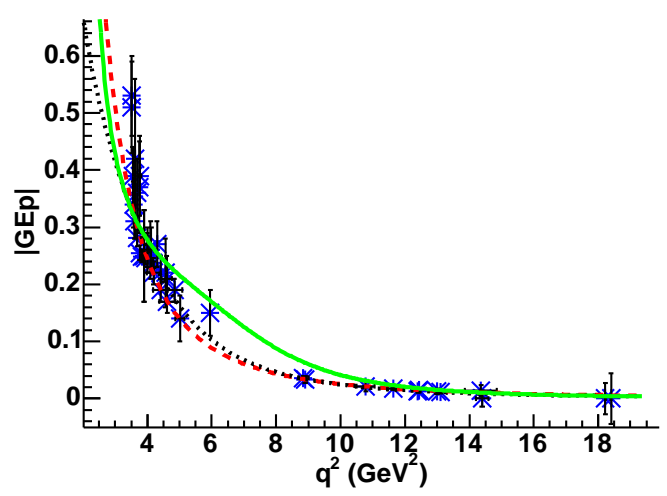

d

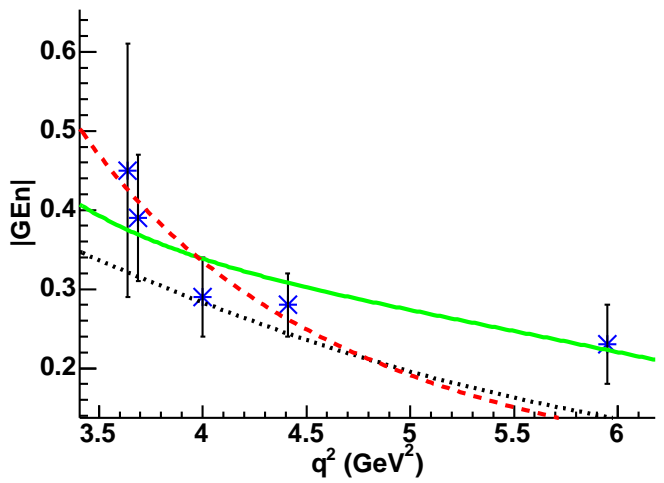

FIG. 4: Form Factors in Time-Like region and predictions of the models: pQCD-inspired (dashed line), from Ref. [62] (dotted line), from Ref. [63] (solid line).

[4] An International Accelerator Facility for Beams of Ions and Antiprotons, Conceptual Design Report, http://www.gsi.de

[5] M. K. Jones et al. [Jefferson Lab Hall A Collaboration], Phys. Rev. Lett. 84, 1398 (2000).

[6] O. Gayou et al., Phys. Rev. Lett. 88, 092301 (2002).

[7] G. Warren et al. [Jefferson Lab E93-026 Collaboration], Phys. Rev. Lett. 92, 042301 (2004);

[8] R. Madey et al. [E93-038 Collaboration], Phys. Rev. Lett. 91, 122002 (2003).

[9] D. I. Glazier et al., arXiv:nucl-ex/0410026.

[10] JLab Proposal 04-108: 'Measurement of $G_{E p} / G_{M p}$ to $Q^{2}=9 \mathrm{GeV}^{2}$ via Recoil Polarization', (Spokepersons: C.F. Perdrisat, V. Punjabi, M.K. Jones and E. Brash), JLab, July 2004.

[11] D. Bisello et al., Nucl. Phys. B 224, 379 (1983).

[12] G. Bardin et al., Nucl. Phys. B 411, 3 (1994).

[13] L. Andivahis et al., Phys. Rev. D 50, 5491 (1994).

[14] S. M. Bilenkii, C. Giunti and V. Wataghin, Z. Phys. C59, 475 (1993).

[15] E. Tomasi-Gustafsson and M. P. Rekalo, Phys. Lett. B 504, 291 (2001) and refs herein.

[16] S. J. Brodsky, C. E. Carlson, J. R. Hiller and D. S. Hwang, Phys. Rev. D 69, 054022 (2004).

[17] F. Iachello, eConf C0309101, FRWP003 (2003), [arXiv:nucl-th/0312074].

[18] F. Iachello and Q. Wan, Phys. Rev. C 69, 055204 (2004).

[19] S. Dubnicka, A. Z. Dubnickova, and P. Weisenpacher, J. Phys. G. 29, 405 (2003); S. Dubnicka, A. Z. Dubnickova,arXiv:hep$\mathrm{ph} / 0401081$.

[20] R. Bijker and F. Iachello, Phys. Rev. C 69, 068201 (2004).

[21] A. Zichichi, S.M. Berman, N. Cabibbo and R. Gatto, Nuovo Cimento XXIV, 170 (1962).

[22] M. P. Rekalo and E. Tomasi-Gustafsson, Eur. Phys. J. A 22, 331 (2004) and refs herein.

[23] V. Punjabi et al., arXiv:nucl-ex/0501018.

[24] W. Albrecht, H. J. Behrend, F. W. Brasse, W. Flauger, H. Hultschig and K. G. Steffen, Phys. Rev. Lett. 17, 1192 (1966).

[25] T. Janssens et al., Phys. Rev. 142, 922 (1966).

[26] D. H. Coward et al., Phys. Rev. Lett. 20, 292 (1968). 
a

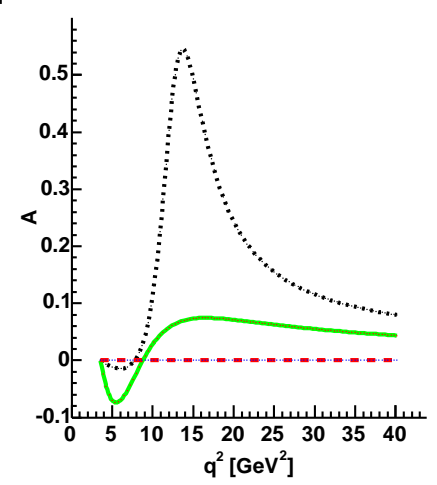

d

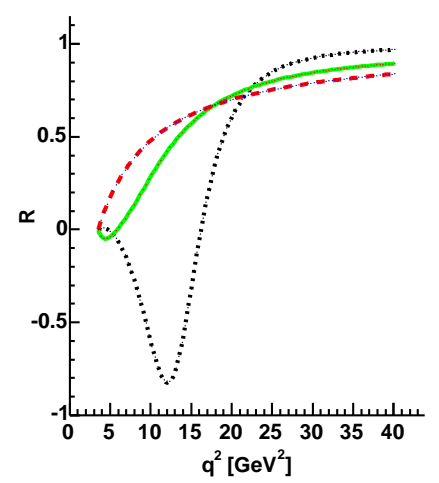

b

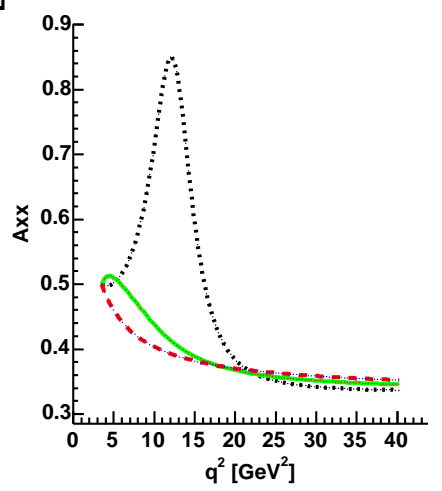

曰

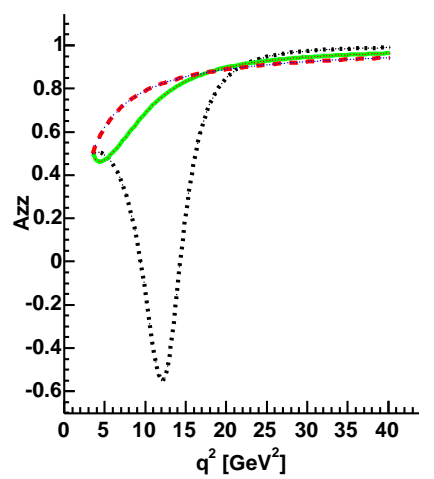

c

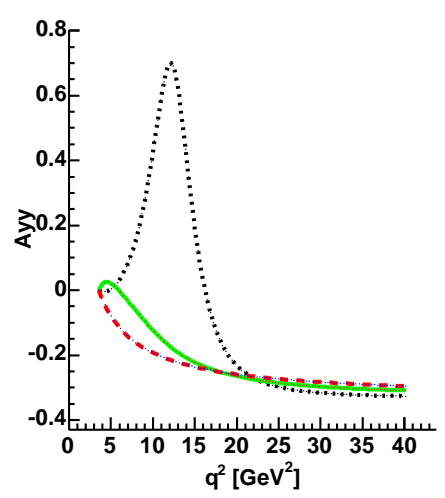

f

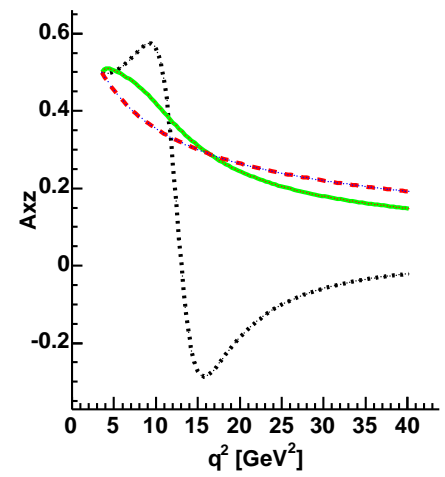

FIG. 5: Angular asymmetry and polarization observables, according to Eqs. (13) and (14), for a fixed value of $\theta=45^{\circ}$. Notations as in Fig. 4.

[27] C. Berger, V. Burkert, G. Knop, B. Langenbeck and K. Rith, Phys. Lett. B 35, 87 (1971).

[28] W. Bartel et al., Nucl. Phys. B 58, 429 (1973).

[29] P. N. Kirk et al., Phys. Rev. D 8, 63 (1973).

[30] A. F. Sill et al., Phys. Rev. D 48, 29 (1993).

[31] R. C. Walker et al., Phys. Rev. D 49, 5671 (1994).

[32] M. E. Christy et al. [E94110 Collaboration], Phys. Rev. C 70, 015206 (2004)

[33] B. D. Milbrath et al. [Bates FPP collaboration], Phys. Rev. Lett. 80, 452 (1998) [Erratum-ibid. 82, 2221 (1999)].

[34] I. A. Qattan et al., arXiv:nucl-ex/0410010.

[35] E.B. Hughes et al., Phys. Rev. 139 (1965) B458, Phys. Rev 146, 973 (1966).

[36] P. Stein, M. Binkley, R. McAllister, A. Suri and W. Woodward, Phys. Rev. Lett. 16, 592 (1966).

[37] W. Bartel et al., Phys. Lett. B 30, 285 (1969).

[38] K. M. Hanson, J. R. Dunning, M. Goitein, T. Kirk, L. E. Price and R. Wilson, Phys. Rev. D 8, 753 (1973).

[39] S. Rock et al., Phys. Rev. Lett. 49, 1139 (1982).

[40] C. E. Jones-Woodward et al., Phys. Rev. C 44, 571 (1991).

[41] C.W. Akerlof et al., Phys. Rev. 135, 810 (1964).

[42] T. Eden et al., Phys. Rev. C 50, 1749 (1994).

[43] M. Meyerhoff et al., Phys. Lett. B 327 (1994) 201.

[44] E. E. W. Bruins et al., Phys. Rev. Lett. 75, 21 (1995).

[45] H. Anklin et al., Phys. Lett. B 428, 248 (1998).

[46] J. Becker et al., Eur. Phys. J. A 6, 329 (1999).

[47] C. Herberg et al., Eur. Phys. J. A 5, 131 (1999).

[48] M. Ostrick et al., Phys. Rev. Lett. 83, 276 (1999).

[49] I. Passchier et al., Phys. Rev. Lett. 82, 4988 (1999).

[50] J. Bermuth et al., Phys. Lett. B 564, 199 (2003).

[51] G. Warren et al. [Jefferson Lab E93-026 Collaboration], Phys. Rev. Lett. 92, 042301 (2004). 
[52] J. Golak, G. Ziemer, H. Kamada, H. Witala and W. Gloeckle, Phys. Rev. C 63, 034006 (2001).

[53] H. Zhu et al. [E93026 Collaboration], Phys. Rev. Lett. 87, 081801 (2001).

[54] M. Castellano et al., Nuovo Cim. A 14, 1 (1973).

[55] G. Bassompierre et al., Phys. Lett. B 68, 477 (1977).

[56] G. Bassompierre et al., Nuovo Cim. A 73, 347 (1983).

[57] B. Delcourt et al., Phys. Lett. B 86, 395 (1979).

[58] T. A. Armstrong et al. [E760 Collaboration], Phys. Rev. Lett. 70, 1212 (1993).

[59] A. Antonelli et al., Phys. Lett. B 334, 431 (1994).

[60] M. Ambrogiani et al. [E835 Collaboration], Phys. Rev. D 60, 032002 (1999).

[61] A. Antonelli et al., Nucl. Phys. B 517, 3 (1998).

[62] F. Iachello, A. D. Jackson and A. Lande, Phys. Lett. B 43, 191 (1973).

[63] E. L. Lomon, Phys. Rev. C 66, 045501 (2002).

[64] G. Hohler, E. Pietarinen, I. Sabba Stefanescu, F. Borkowski, G. G. Simon, V. H. Walther and R. D. Wendling, Nucl. Phys. B 114, 505 (1976).

[65] P. E. Bosted, Phys. Rev. C 51, 409 (1995).

[66] G. P. Lepage and S. J. Brodsky, Phys. Rev. D 22, 2157 (1980); Phys. Rev. Lett. 43, 545 (1979) [Erratum-ibid. 43, 1625 (1979)].

[67] P. Gauzzi, Phys. Atom. Nucl.59, 1382 (1996) [Yad. Fiz. 59, 1441 (1996)].

[68] S. Galster, H. Klein, J. Moritz, K. H. Schmidt, D. Wegener and J. Bleckwenn, Nucl. Phys. B 32, 221 (1971).

[69] A. Z. Dubnickova, S. Dubnicka and M. P. Rekalo, Nuovo Cim. A 109, 241 (1996).

[70] M. P. Rekalo, Sov. J. Nucl. Phys. 1, 760 (1965) [J. Nucl. Phys. (USSR) 1, 1066 (1965)].

[71] A. Z. Dubnickova, S. Dubnicka and M. P. Rekalo, Z. Phys. C 70, 473 (1996).

\begin{tabular}{|c|c|c|c|c|c|}
\hline Reaction & $Q^{2}\left[\mathrm{GeV}^{2}\right]$ & Observables & Laboratory & Year & Reference \\
\hline$e+p \rightarrow e+p$ & $4.08-9.59$ & $G_{M p}$ & DESY & 1966 & Albrecht et al. [24] \\
\hline$"$ & $0.16-0.85$ & $G_{E p}, G_{M p}$ & SLAC & 1966 & Janssens et al. [25] \\
\hline$"$ & $0.69-25.03$ & $G_{M p}$ & SLAC & 1968 & Coward et al. [26] \\
\hline$"$ & $0.4-2$ & $G_{E p}, G_{M p}$ & Bonn & 1971 & Berger et al. [27] \\
\hline$"$ & $0.670-3.01$ & $G_{E p}, G_{M p}$ & DESY & 1973 & Bartel et al. [28] \\
\hline & $0.999-25.03$ & $G_{M p}$ & SLAC & 1973 & Kirk et al. [29] \\
\hline$"$ & $2.86-31.2$ & $G_{M p}$ & SLAC & 1993 & Sill et al. [30] \\
\hline$"$ & $1-3$ & $G_{E p}, G_{M p}$ & SLAC & 1994 & Walker et al. [31] \\
\hline$"$ & $1.75-8.83$ & $G_{M p}$ & SLAC & 1994 & Andivahis et al.[13] \\
\hline$"$ & $0.65-5.20$ & $G_{E p}, G_{M p}$ & JLab, E94110 & 2004 & Christy et al. [32] \\
\hline$"$ & $0.65-5.20$ & $G_{E p}, G_{M p}$ & JLab, Hall A & 2004 & Qattan et al. [34] \\
\hline$\vec{e}+p \rightarrow e+\vec{p}$ & $0.49-3.47$ & $G_{E p} / G_{M p}$ & JLAB, Hall A & 2000 & Jones et al. [5] \\
\hline$\vec{e}+p \rightarrow e+\vec{p}$ & $3.5-5.5$ & $G_{E p} / G_{M p}$ & JLAB, Hall A & 2002 & Gayou et al. [6] \\
\hline
\end{tabular}

TABLE I: Data considered in the present analysis, for proton FFs, in SL region. 


\begin{tabular}{|c|c|c|c|c|c|}
\hline Reaction & $Q^{2}\left[\mathrm{GeV}^{2}\right]$ & Observables & Laboratory & Year & Reference \\
\hline$e d \rightarrow e p n$ & $0.04-1.16$ & $G_{E n}$ & SLAC & 1965 & Hughes et al. [35] \\
\hline$e d \rightarrow e p n$ & $0.19,0.39,0.56$ & $G_{M n}$ & New York & 1966 & Stein et al. [36] \\
\hline$e d \rightarrow e p n$ & $0.39-0.565$ & $G_{M n}, G_{E n}$ & DESY & 1969 & Bartel et al. [37] \\
\hline$e d \rightarrow e p n$ & $0.28-1.8$ & $G_{M n}$ & Harvard & 1973 & Hanson et al. [38] \\
\hline$e d \rightarrow e p n$ & $2.5-10$ & $G_{M n}$ & SLAC & 1982 & Rock et al. [39] \\
\hline${ }^{3} \overrightarrow{H e}$ & 0.16 & $G_{E n}$ & MIT & 1991 & Jones-Woodward et al. [40] \\
\hline$e d \rightarrow$ epn & $0.435-1.36$ & $G_{M n}$ & New York & 1964 & Akerlof et al. [41] \\
\hline$\vec{D}\left(\vec{e}, e^{\prime} \vec{n}\right) p$ & .0 .255 & $G_{E n}$ & MIT & 1994 & Eden et al. [42] \\
\hline$D\left(e, e^{\prime} n\right), D\left(e, e^{\prime} p\right)$ & $0.125-0.605$ & $G_{E n}$ & Bonn & 1995 & Bruins et al. [44] \\
\hline$D\left(e, e^{\prime} n\right), D\left(e, e^{\prime} p\right)$ & $0.235-0.784$ & $G_{E n}$ & MAMI & 1998 & H. Anklin et al. [45] \\
\hline$D\left(\vec{e}, e^{\prime} \vec{n}\right) p$ & 0.15 & $G_{E n}$ & MAMI & 1999 & Herberg et al. [47] \\
\hline$D\left(\vec{e}, e^{\prime} \vec{n}\right) p$ & 0.34 & $G_{E n}$ & MAMI & 1999 & Ostrick et al. [48] \\
\hline$\vec{D}\left(\vec{e}, e^{\prime} n\right) p$ & 0.21 & $G_{E n}$ & NIKHEF & 1999 & Passchier et al. [49] \\
\hline${ }^{3} \overrightarrow{H e}\left(\vec{e}, e^{\prime} n\right) p p$ & 0.67 & $G_{E n}$ & MAMI & 2003 & Bermuth et al. [50] \\
\hline${ }^{3} \overrightarrow{H e}\left(\vec{e}, e^{\prime}\right)$ & $0.1-0.4$ & $G_{E n}, G_{M n}$ & JLab & 2000 & Golak et al.[52] \\
\hline$\vec{D}\left(\vec{e}, e^{\prime} n\right) p$ & 0.495 & $G_{E n}$ & JLab & 2001 & Zhu et al. [53] \\
\hline$\vec{D}\left(\vec{e}, e^{\prime} n\right) p$ & $0.5,1$ & $G_{E n}$ & JLab & 2004 & Warren et al. [7] \\
\hline$D\left(\vec{e}, e^{\prime} \vec{n}\right) p$ & $0.3-0.8$ & $G_{E n}$ & MAMI & 2004 & Glazier et al. [9] \\
\hline$D\left(\vec{e}, e^{\prime} \vec{n}\right) p$ & $0.5-1.5$ & $G_{E n}$ & JLab & 1999 & Madey et al. [8] \\
\hline
\end{tabular}

TABLE II: Data considered in the present analysis, for neutron FFs, in SL region.

\begin{tabular}{|c|c|c|l|l|}
\hline Reaction & $q^{2}\left[\mathrm{GeV}^{2}\right]$ & Laboratory & Year & Reference \\
\hline$e^{+} e^{-} \rightarrow p \bar{p}$ & 4.3 & ADONE, Frascati & 1973 & Castellano et al. $[54]$ \\
$p \bar{p} \rightarrow e^{+} e^{-}$ & 3.52 & CERN & 1977 & Bassompierre et al. $[55]$ \\
$p \bar{p} \rightarrow e^{+} e^{-}$ & 3.61 & CERN & 1983 & Bassompierre et al. $[56]$ \\
$e^{+} e^{-} \rightarrow p \bar{p}$ & $3.75-4.56$ & Orsay,DCI & 1979 & Delcourt et al. $[57]$ \\
$e^{+} e^{-} \rightarrow p \bar{p}$ & $4.0-5.0$ & Orsay, DCI & 1983 & Bisello et al. $[11]$ \\
$p \bar{p} \rightarrow e^{+} e^{-}$ & $8.9-13.0$ & FERMILAB, E760 & 1993 & Armstrong et al. [58] \\
$p \bar{p} \rightarrow e^{+} e^{-}$ & $3.52-4.18$ & CERN, LEAR & 1994 & Bardin et al. $[12]$ \\
$e^{+} e^{-} \rightarrow p \bar{p}$ & $3.69-5.95$ & ADONE, FENICE & 1994 & Antonelli et al. [59] \\
$p \bar{p} \rightarrow e^{+} e^{-}$ & $8.84-18.40$ & FERMILAB, E835 & 1999 & Ambrogiani et al. $[60]$ \\
$p \bar{p} \rightarrow e^{+} e^{-}$ & $11.63-18.22$ & FERMILAB, E835 & 2003 & Andreotti et al. $[2]$ \\
\hline$e^{+} e^{-} \rightarrow n \bar{n}$ & $3.61-5.95$ & ADONE, FENICE & 1998 & Antonelli et al. $[45]$ \\
\hline
\end{tabular}

TABLE III: Data considered in the present analysis for TL region. 


\begin{tabular}{|c|c|c|}
\hline Parameters & GKex(02L) & This work \\
\hline$g_{\rho^{\prime}} / f_{\rho^{\prime}}$ & 0.0608 & -0.0152 \\
$\kappa_{\rho^{\prime}}$ & 5.3038 & -30.315 \\
$g_{\omega} / f_{\omega}$ & 0.6896 & 0.5994 \\
$\kappa_{\omega}$ & -2.8585 & 5.4557 \\
$g_{\phi} / f_{\phi}$ & -0.1852 & -0.287304 \\
$\kappa_{\phi}$ & 13.0037 & 18.0208 \\
$\mu_{\phi}$ & 0.6848 & 0.802158 \\
$g\left(\omega^{\prime}\right) / f_{\rho^{\prime}}$ & 0.2346 & 0.125397 \\
$k\left(\omega^{\prime}\right)$ & 18.2284 & 15.4868 \\
$\Lambda_{1}$ & 0.9441 & 0.776982 \\
$\Lambda_{D}$ & 1.2350 & 1.06593 \\
$\Lambda_{2}$ & 2.8268 & 3.64885 \\
$\Lambda_{Q C D}$ & 0.150 & 0.377101 \\
$N$ & 1. & 0.84501 \\
\hline
\end{tabular}

TABLE IV: Parameters of the model from [63]. Fixed constants are $\kappa_{v}=3.706, \kappa_{s}=-0.12, m_{\rho}=0.776 \mathrm{GeV}, m_{\omega}=$ $0.784 \mathrm{GeV}, m_{\phi}=1.019 \mathrm{GeV}, m_{\rho^{\prime}}=1.45 \mathrm{GeV}, m_{\omega^{\prime}}=1.419 \mathrm{GeV}$. 


\begin{tabular}{|c|c|c|}
\hline Parameters & {$[18,62]$} & This work \\
\hline \hline$\gamma$ & 0.250 & 0.259 \\
$\beta_{\rho}$ & 0.672 & 0.757 \\
$\beta_{\omega}$ & 1.102 & 1.212 \\
$\beta_{\phi}$ & 0.112 & -0.114 \\
$\alpha_{\phi}$ & -0.052 & -0.028 \\
$\theta$ & $53^{0}$ & $47^{0}$ \\
\hline
\end{tabular}

TABLE V: Parameters for the model from Ref. [18, 62].

\begin{tabular}{|c|c|c|c||c|c|c|c|}
\hline FFs & par & {$[64]$} & This work & FFs & par & {$[64]$} & This work \\
\hline \hline$F_{i s}$ & $b_{1}^{(s)}$ & 0.61 & 0.49 & $F_{i v}$ & $b_{1}^{(v)}$ & 1.49 & 1.35 \\
& $b_{2}^{(s)}$ & 0.94 & 0.84 & & $b_{2}^{(v)}$ & 4.33 & 3.22 \\
& $b_{3}^{(s)}$ & 3.20 & 0.47 & & $b_{3}^{(v)}$ & 8.47 & 21.28 \\
\hline \multirow{2}{*}{$F_{1 s}$} & $a_{1}^{(1, s)}$ & 0.75 & 0.74 & $F_{1 v}$ & $a_{1}^{(1, v)}$ & 0.06 & 0.23 \\
& $a_{2}^{(1, s)}$ & -0.61 & -0.57 & & $a_{2}^{(1, v)}$ & -0.32 & -0.55 \\
& $a_{3}^{(1, s)}$ & -0.23 & -0.13 & & $a_{3}^{(1, v)}$ & 0.10 & 0.04 \\
\hline \multirow{2}{*}{$F_{2 s}$} & $a_{1}^{(2, s)}$ & -0.15 & 0.74 & $F_{2 v}$ & $a_{1}^{(2, v)}$ & -2.06 & -1.93 \\
& $a_{2}^{(2, s)}$ & 0.18 & -0.57 & & $a_{2}^{(2, v)}$ & 0.23 & 0.22 \\
& $a_{3}^{(2, s)}$ & -0.03 & -0.13 & & $a_{3}^{(2, v)}$ & 0.23 & 0.13 \\
\hline
\end{tabular}

TABLE VI: Parameters for the model from Ref. [64], corresponding to the fit in SL region, only. The starting set of parameters is from fit No. 9.1

\begin{tabular}{|c|c|c|c|}
\hline $\mathrm{FF}$ & $a_{i}^{(j)}$ & {$[65]$} & This work \\
\hline \hline$G_{E p}$ & $a_{1}^{1}$ & 0.62 & -1.55 \\
& $a_{2}^{1}$ & 0.68 & 10.40 \\
& $a_{3}^{1}$ & 2.80 & -11.53 \\
& $a_{4}^{1}$ & 0.83 & 7.87 \\
\hline$G_{M p} / \mu_{p}$ & $a_{1}^{2}$ & 0.35 & 0.28 \\
& $a_{2}^{2}$ & 2.44 & 2.61 \\
& $a_{3}^{2}$ & 1.04 & 0.41 \\
& $a_{4}^{2}$ & 0.34 & 1.03 \\
& $a_{5}^{2}$ & 0.34 & 0.34 \\
\hline$G_{M n} / \mu_{n}$ & $a_{1}^{3}$ & -1.74 & 0.14 \\
& $a_{2}^{3}$ & 9.29 & 3.39 \\
& $a_{3}^{3}$ & -7.63 & -2.07 \\
& $a_{4}^{3}$ & 4.63 & 3.09 \\
\hline$G_{E n}$ & $\alpha$ & 1.25 & 0.43 \\
& $\beta$ & 18.3 & -0.22 \\
\hline
\end{tabular}

TABLE VII: Parameters for the model from Ref. [65], corresponding to the fit in SL region, only. 